\title{
CHILI, a Nanobeam Secondary Neutral Mass Spectrometer with Extraordinary Spatial Resolution, Sensitivity, and Selectivity: First Results.
}

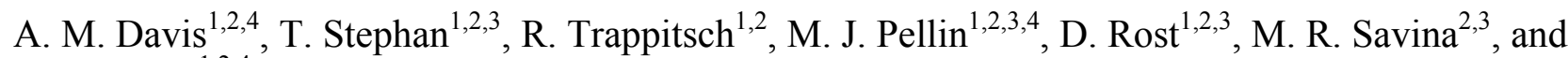 \\ N. Dauphas ${ }^{1,2,4}$. \\ ${ }^{1}$ Department of the Geophysical Sciences, The University of Chicago, Chicago, IL 60637, USA. \\ ${ }^{2}$ Chicago Center for Cosmochemistry. \\ ${ }^{3}$ Materials Science Division, Argonne National Laboratory, Argonne, IL 60439, USA. \\ ${ }^{4}$ The Enrico Fermi Institute, The University of Chicago, Chicago, IL 60637, USA.
}

CHILI, the Chicago Instrument for Laser Ionization, is a nanobeam secondary neutral mass spectrometer using laser resonance ionization that is designed for a lateral resolution as small as $10 \mathrm{~nm}$, a useful yield (atoms detected per atom desorbed) of 30-50\%, and nearly complete suppression of isobaric interferences from both monatomic and molecular ions [1 and references therein]. It is equipped with an Orsay Physics Cobra liquid metal ion gun with a lateral resolution of $2.5 \mathrm{~nm}$ for desorbing from small spots and rastered areas (less than a few $\mu \mathrm{m}$ ) and a $351 \mathrm{~nm}$ laser for desorbing from spots or rastered areas from the $\mu \mathrm{m}$ scale upwards. An optical microscope is built into CHILI and is used to image the sample and introduce the ablation laser. An Orsay Physics e CLIPSE-plus field emission electron gun with a resolution of $4 \mathrm{~nm}$ is also used for imaging samples. CHILI is equipped with six tunable Ti:sapphire lasers that we designed and built, pumped by three Photonics DM-40 $40 \mathrm{~W} \mathrm{Nd:YLF} \mathrm{lasers}$ $(527 \mathrm{~nm})$ for simultaneous isotopic measurement of two or three elements. The Ti:sapphire lasers are tunable from $\sim 700-1000 \mathrm{~nm}$, but can be frequency doubled $(350-500 \mathrm{~nm})$, tripled $(233-333 \mathrm{~nm})$ or quadrupled $(205-250 \mathrm{~nm})$ with nonlinear optical crystals. We anticipate a lateral resolution of $\sim 10 \mathrm{~nm}$ for resonance ionization using the $\mathrm{Ga}^{+}$primary beam, as this is the approximate analytical volume for sputtering; for laser ablation, the minimum spot size will be $\sim 1 \mu \mathrm{m}$. CHILI is housed within a dedicated laboratory with controlled temperature and humidity. The analytical sequence is the following: release atoms from the surface by sputtering; eject secondary ions by applying high accelerating voltage to the cloud; apply the normal accelerating voltage; fire the photoionization lasers; and mass analyze the photoions. CHILI's removable sample holder is capable of mounting a variety of common types of samples, including 1" diameter polished sections, 1/2" and $1 \mathrm{~cm}$ diameter SEM stubs, and TEM samples.

As an initial test of CHILI, we analyzed some presolar SiC grains from mount KJG\#2. The grains were extracted from the Murchison CM2 meteorite more than 20 years ago [2] and represent samples of stars that lived and died before the solar system formed. In contrast to recent work on KJG grains [3-5], the samples in this study were not additionally treated with concentrated acids to remove parent-body or terrestrial contamination. The grains were mounted on a high purity gold foil by depositing them from a suspension and pressing them into the gold with a sapphire window. Prior to analysis, energy dispersive $\mathrm{X}$-ray images of the mount were acquired in a scanning electron microscope to locate $\mathrm{SiC}$ grains on the gold foil; 22 grains were selected for this study. Using previously developed ionization schemes, the Ti:sapphire lasers were tuned for resonance ionization of strontium, zirconium, and barium. Isotopic standards used in this study are NIST SRM 855a with 180 ppm Sr, Zr metal, NIST SRM 1264a with $690 \mathrm{ppm} \mathrm{Zr}$, and terrestrial $\mathrm{BaTiO}_{3}$, all of which were assumed to be of normal terrestrial isotopic composition. Data were corrected for dead time effects; the detector dead time was found to be $600 \mathrm{ps,}$ which was determined by analyzing $\mathrm{Zr}$ metal by ion sputtering with varying primary ion beam current. Instrumental isotopic fractionation was determined to be smaller than the statistical error of typically a 
few tens of \%o except for ${ }^{91} \mathrm{Zr}$, which showed enhanced laser resonance ionization by $\sim 210 \%$ due to an odd-even effect as had been previously observed [6] and which is corrected for by analyzing standards.

Ten grains had sufficient strontium and/or barium content for isotopic analysis with CHILI. However, none of the grains had detectable zirconium. Strontium and barium isotopic ratios, normalized to ${ }^{86} \mathrm{Sr}$ and ${ }^{136} \mathrm{Ba}$, respectively, are displayed in Fig. 1 as $\delta$ values (deviation from standards in \%o). For comparison, Fig. 1 shows data obtained with an earlier generation instrument (CHARISMA) on SiC grains that had undergone acid cleaning [3-5]. Most of the SiC grains analyzed in the present study show a similar range of isotope ratios as in the previous studies, and are consistent with formation in asymptotic giant branch stars [3-5]. There seems to be a tendency to plot closer to normal than the previously analyzed grains; residual parent-body or terrestrial contamination could be responsible for such a trend. One of the grains has substantial excesses in ${ }^{88} \mathrm{Sr}$ and ${ }^{138} \mathrm{Ba}$. This grain is likely from a core-collapse supernova, and the pattern is due to a neutron burst shortly after the supernova explosion. Two grains show significant depletions in ${ }^{87} \mathrm{Sr}$ and ${ }^{88} \mathrm{Sr}$, patterns that have not yet been explained.

A wide variety of cosmochemical problems will be explored with CHILI, including isotopic and chemical studies of presolar grains, refractory inclusions from meteorites, samples of the Sun returned to Earth by the Genesis spacecraft, and cometary and interstellar dust returned by the Stardust spacecraft.

References

[1] T Stephan et al. Lunar Planet. Sci. 45 (2014), \#2242.

[2] S Amari et al. Geochim. Cosmochim. Acta 58 (1994), 459.

[3] N Liu et al. Astrophys. J. 786 (2014), \#66.

[4] N Liu et al. Astrophys. J. 788 (2014), \#163.

[5] N Liu Dissertation, The University of Chicago (2014), 181 pp.

[6] GK Nicolussi et al. Science 277 (1997), 1281.
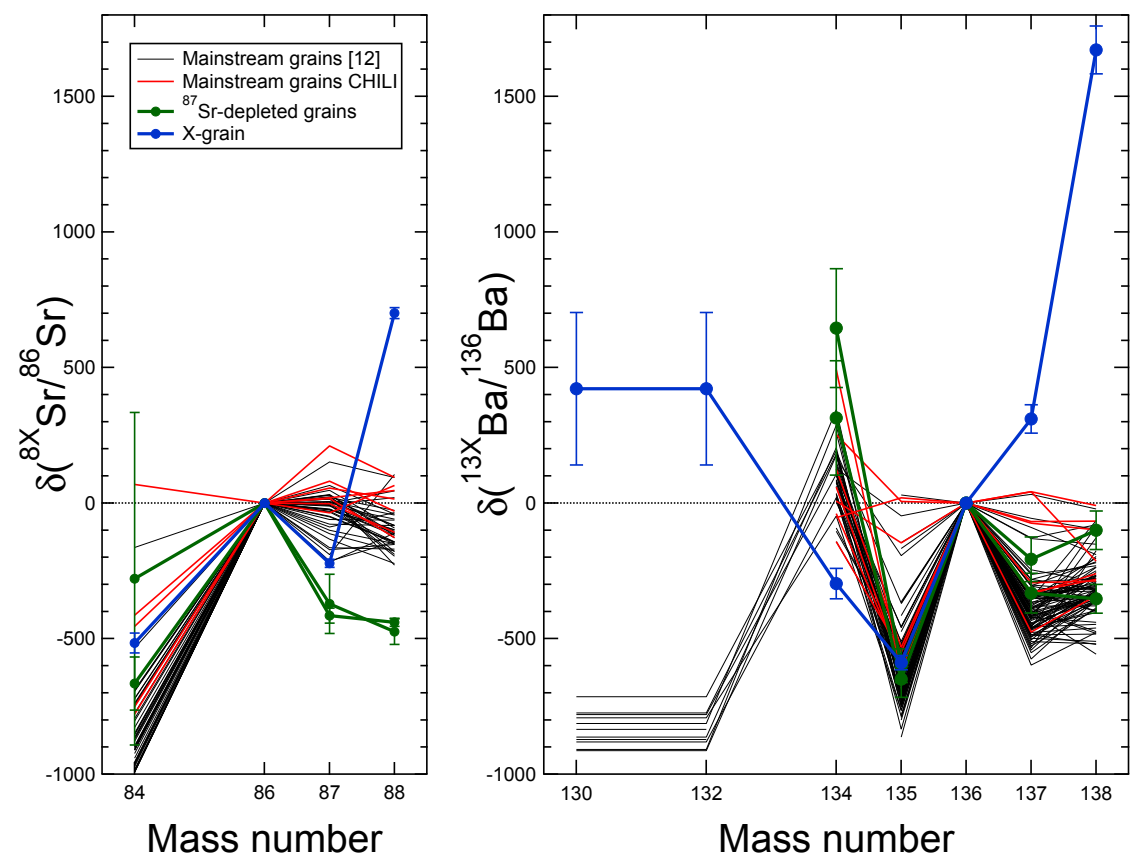

Figure 1. $\mathrm{Sr}$ and $\mathrm{Ba}$ isotopic patterns of presolar $\mathrm{SiC}$ grains. Error bars are $\pm 2 \sigma$. 